DFF 354/05/2000

hep-th/0005115

\title{
On the Trace Anomaly as a Measure of Degrees of Freedom
}

\author{
Andrea CAPPELLI and Giuseppe D'APPOLLONIO \\ I.N.F.N. and Dipartimento di Fisica \\ Largo E. Fermi 2, I-50125 Firenze, Italy
}

\begin{abstract}
Recent conjectures of the $c$-theorem in four and higher dimensions have suggested that the coefficient of the Euler characteristic in the trace anomaly could measure the degrees of freedom in field theory and decrease along the renormalization-group flow. We compute this quantity for free massless scalar, fermion and antisymmetric tensor fields in any dimension, and analyse its dependence on spin and space-time dimension. In the limit of large number of dimensions, where the theories become semiclassical, we find that this quantity does not approach the classical number of field components, but is enhanced for spinful particles. This seemingly strange behaviour is found to be consistent with known renormalization-group patterns and a specific $c$-theorem conjecture.
\end{abstract}

May 2000 


\section{Introduction}

The $c$-theorem establishes the irreversibility of the renormalization-group flow in two dimensions [1] ; it shows that there exists a positive function of the coupling constants which monotonically decreases along the flows and is stationary at the fixed points. The existence of an analogous theorem in four and higher dimensions [2] [3] has been extensively discussed in the recent literature: several non-trivial flows have been checked in four-dimensional supersymmetric gauge theories (most notably in the "conformal window") [4], and via the AdS/CFT correspondence [5]; moreover, two proofs of the theorem have been presented in the Refs. [6] [7].

Most of the examples and conjectures identify the $c$-function at the fixed points with the coefficient of the Euler density $G$ in the trace anomaly (such that $\chi=\int G$ is the Euler characteristic). This coefficient, called $a$, has definite sign for all unitary theories [8], and verify the inequality $a_{U V}>a_{I R}$ for all known examples of flows connecting pairs of UV and IR fixed points.

According to the analysis of Ref. [9], the trace anomaly in any even dimension $d=2 k$ contains three parts: the topological invariant term, given by the Euler density; the Weyl invariant terms, made by polynomials of the Weyl tensor and its derivatives, and finally the variations of local counterterms which can be neglected. On conformallyflat geometries, like the $d$-dimensional sphere $S^{2 k}$, the Weyl tensor vanishes and the trace anomaly is completely expressed in terms of the Euler characteristic.

In this paper we calculate the trace anomaly on the sphere $S^{2 k}$ for the free massless scalar, Dirac spinor and antisymmetric tensor fields in any dimension and obtain the corresponding values of $a$. We use the standard zeta-function regularization of the partition function [10].

Next, we analyse the behaviour of $a$ upon varying the spin and the dimension and test its interpretation as a measure of degrees of freedom in field theory. In the large- $d$ limit, we find that all anomalies, once properly normalized, go to zero; this is consistent with the expected semiclassical behaviour. However, the ratios $a(\sigma) / a(0)$ of the spinful to the scalar values, which have the meaning of counting functions, do not approach the classical number of fields components: each component of the antisymmetric tensor field weights of order $O\left(d^{3}\right)$ with respect to the scalar field, and each fermion component weights $O(d)$. Therefore, the anomaly a yields a "quantum" measure of degrees of freedom which is rather far from the classical intuition, with 
the antisymmetric tensor dominating the counting for any $d \geq 4$.

At first sight, we could doubt of this interpretation as a measure of degrees of freedom; however, a closer inspection shows that this is consistent with known facts and renormalization-group flows. The same enhancement is found for the gravitational chiral anomaly [11] and for another term in the trace anomaly, whose coefficient $c$ also normalizes the stress-tensor two-point function [3]. Furthermore, the observed dominance of the antisymmetric tensor field is crucial for the existence of theories with $c \propto a$ in any dimension [12]; these theories naturally arise in the AdS/CFT correspondence [13] 14 and have been conjectured to satisfy a version of $c$-theorem with all its two-dimensional features [12].

\section{Trace anomaly of the scalar and Dirac fermion fields}

The stress tensor $T_{\mu \nu}$ of a $d$-dimensional field theory is defined by the variation of the partition function with respect to the the background metric $g_{\mu \nu}$ :

$$
\delta \log Z\left[g_{\mu \nu}\right]=\frac{1}{2 V_{d-1}} \int d x^{d} \sqrt{g} T_{\mu \nu} \delta g^{\mu \nu}, \quad V_{d-1}=\frac{2 \pi^{d / 2}}{\Gamma\left(\frac{d}{2}\right)},
$$

where the conventional normalization factor $V_{d-1}$ is the volume of the unit sphere $S^{d-1}$. We shall compute the partition function of $d$-dimensional free field theories on the sphere $S^{2 k}(d=2 k)$ and obtain the integrated trace anomaly by the scale variation $g_{\mu \nu} \rightarrow \exp (2 \alpha) g_{\mu \nu} \quad(\alpha=$ const. $)$ :

$$
\frac{d}{d \alpha} \log Z\left[S^{2 k}\right]=-\frac{1}{V_{2 k-1}} \int_{S^{2 k}}\langle\Theta\rangle=-\frac{V_{2 k} r^{2 k}}{V_{2 k-1}}\langle\Theta\rangle
$$

In this Equation, $\Theta$ is a short-hand notation for the trace $T_{\mu}^{\mu}$ and $r$ is the radius of the sphere.

The (Euclidean) action for bosonic free fields is written in terms of the covariant Laplacian $\Delta$, whose explicit form depends on the spin of the field:

$$
S[g, \phi]=\frac{1}{2} \int \sqrt{g} \phi(x) \Delta \phi(x) .
$$

\footnotetext{
${ }^{*} c$ is always positive, but is not always decreasing [3] [4].
} 
The partition function, i.e. the determinant of the Laplacian, can be obtained from the analytic continuation of the zeta-function [10 associated to the spectrum of $\Delta$ on $S^{2 k}$

$$
\zeta_{\Delta}(s)=\sum_{n=0}^{\infty} \frac{\delta_{n}}{\lambda_{n}^{s}}
$$

this function is defined in terms of the eigenvalues $\lambda_{n}$ and their degeneracies $\delta_{n}$.

We consider theories which are invariant under conformal (Weyl) transformations $g_{\mu \nu}(x) \rightarrow \exp 2 \sigma(x) g_{\mu \nu}(x)$ at the classical level [15] 16]; the corresponding Laplacian is conformally covariant, $\Delta \rightarrow \exp (-(\delta+2) \sigma) \Delta \exp (\delta \sigma)$, with $\delta$ the dimension of $\phi$. In these theories, the scale dependence of the partition function (2.2) is purely anomalous, i.e. is induced by the regularization of the determinant and it is given by the zeta-function analytically continued at $s=0$ [10]:

$$
\frac{d}{d \alpha} \log Z\left[S^{2 k}\right]=\zeta_{\Delta}(0)=-\frac{1}{V_{2 k-1}} \int_{S^{2 k}}\langle\Theta\rangle .
$$

In the case of fermionic fields, we consider the action (2.3) with $\Delta$ the square of the Dirac operator and obtain the anomaly (2.5) in terms of $-\zeta_{\Delta}(0)$.

We now evaluate the trace anomaly for the scalar field Laplacian is [15]:

$$
\Delta_{0}=-\nabla^{2}+\frac{k-1}{2(2 k-1)} \mathcal{R},
$$

with $\mathcal{R}$ the scalar curvature. The eigenvalues and degeneracies of this operator on $S^{2 k}$ are given by

$$
\begin{aligned}
\lambda_{l} & =(l+k)(l+k-1), \quad l=0,1,2, \ldots \\
\delta_{l} & =(2 l+2 k-1) \frac{(l+2 k-2) !}{l !(2 k-1) !}
\end{aligned}
$$

The zeta-function of the scalar Laplacian $\zeta_{\Delta_{0}}(s)(2.4,2.7)$ can be analytically continued at $s=0$ by expressing it in terms of ordinary Riemann zeta-functions $\zeta(s)$. It is convenient to use the analytic continuation formula found in Ref. 18.:

$$
\begin{aligned}
\sum_{\sigma=n+1}^{\infty} \frac{2 \sigma+1}{[(\sigma-n)(\sigma+n+1)]^{s}}= & \frac{1}{\Gamma(s)} \sum_{m=0}^{\infty} \frac{\Gamma(s+m-1)}{\Gamma(m+1)}(2 s+m-2)(2 n+1)^{m} \\
& \times\left[\zeta(2 s+m-1)-\sum_{l=1}^{2 n+1} l^{-2 s-m+1}\right] .
\end{aligned}
$$

\footnotetext{
$\dagger$ This calculation and the following one for the Dirac fermion are not new [17], but we present here rather compact results.
} 
We rewrite the zeta-function of the scalar Laplacian as follows (for $k \geq 2$ ):

$$
\zeta_{\Delta_{0}}(s)=\frac{1}{(2 k-1) !} \sum_{i=1}^{k-1} \alpha_{i} \sum_{\sigma=k-1}^{\infty} \frac{2 \sigma+1}{[\sigma(\sigma+1)]^{s-i}}
$$

in terms of the coefficients $\alpha_{i}$ defined by

$$
\frac{(\sigma+k-1) !}{(\sigma-k+1) !}=\prod_{i=0}^{k-2}[\sigma(\sigma+1)-i(i+1)]=\sum_{i=1}^{k-1} \alpha_{i}[\sigma(\sigma+1)]^{i}, \quad(k \geq 2)
$$

where $\sigma=l+k-1$. We use the analytic continuation (2.8) for all the terms in (2.9), which reduce to finite sums involving the Bernoulli numbers $B_{n}$ in the limit $s=0$ :

$$
\zeta_{\Delta_{0}}(0)=-\frac{1}{(2 k-1) !} \sum_{i=1}^{k-1} \alpha_{i} \sum_{m=0}^{i+1} \frac{i !}{m !(i-m+1) !} B_{2 i+2-m} .
$$

This expression can be cast into the following compact form:

$$
\zeta_{\Delta_{0}}(0)=-\frac{1}{(2 k-1) !} \int_{0}^{B(1+B)} d t \prod_{i=0}^{k-2}[t-i(i+1)], \quad(d=2 k \geq 4),
$$

where it is understood that $B^{n}$ should be replaced with $B_{n}$ after evaluation of the integral. The values of $\zeta_{\Delta_{0}}$ for the lowest dimensions $d=\{4,6, \ldots, 14\}$ are:

$$
\zeta_{\Delta_{0}}(0)=\left\{-\frac{1}{90}, \frac{1}{756},-\frac{23}{113400}, \frac{263}{7484400},-\frac{133787}{20432412000}, \frac{157009}{122594472000}\right\} .
$$

Finally, the trace anomaly of the scalar field is obtained by replacing (2.12) into (2.2) (the result is in agreement with Ref. 17]). We postpone the discussion of these numbers to Section 4 and move to the analogous calculation for the Dirac field.

The spinor Laplacian is obtained from the square of the Dirac operator in the $g_{\mu \nu}$ background,

$$
\Delta_{1 / 2}=-\nabla^{2}=-\nabla^{2}+\frac{\mathcal{R}}{4},
$$

and it is conformally covariant [15]; its eigenvalues and degeneracies on the sphere are [19]:

$$
\begin{aligned}
& \lambda_{l}=(l+k)^{2}, \quad l=0,1,2, \ldots \\
& \delta_{l}=2^{k+1} \frac{(2 k+l-1) !}{l !(2 k-1) !} .
\end{aligned}
$$


The analytic continuation of the corresponding zeta-function is again obtained by splitting it into simpler functions $(k \geq 1)$ :

$$
\zeta_{\Delta_{1 / 2}}(s)=\frac{2^{k+1}}{(2 k-1) !} \sum_{l=0}^{\infty} \frac{(l+2 k-1) !}{l !} \frac{1}{(l+k)^{2 s}}=\frac{2^{k+1}}{(2 k-1) !} \sum_{i=0}^{k-1} \beta_{i} \zeta(2 s-2 i-1),
$$

where the coefficients $\beta_{i}$ are defined by

$$
\frac{(\sigma+k-1) !}{(\sigma-k) !}=\sigma \prod_{i=1}^{k-1}\left(\sigma^{2}-i^{2}\right)=\sum_{i=0}^{k-1} \beta_{i} \sigma^{2 i+1}
$$

with $\sigma=l+k$. The limit $s=0$ can be taken into Eq.(2.17) and the result can be written again in compact form:

$$
\zeta_{\Delta_{1 / 2}}(0)=-\frac{2^{k}}{(2 k-1) !} \int_{0}^{B^{2}} d t \prod_{i=1}^{k-1}\left(t-i^{2}\right), \quad\left(B^{n} \rightarrow B_{n}\right)
$$

(for $k=1$ the integrand is one). The first few values for $d=\{4,6, \ldots, 14\}$ are:

$$
-\zeta_{\Delta_{1 / 2}}(0)=\left\{-\frac{11}{90}, \frac{191}{3780},-\frac{2497}{113400}, \frac{14797}{1496880},-\frac{92427157}{20432412000}, \frac{36740617}{17513496000}\right\} .
$$

\section{Trace anomaly of the antisymmetric tensor field}

Another conformal invariant free theory in $2 k$ dimensions is given by the antisymmetric $(k-1)$-form, called antisymmetric tensor field [20], which generalizes the four-dimensional vector field. Antisymmetric tensor fields commonly appear in supergravity and in superstring theory; a well known example 21] is the low-energy world-volume theory of the $M 5$-brane described by a $d=6, \mathcal{N}=(2,0)$ tensor multiplet that contains one antisymmetric tensor $B_{\mu \nu}$.

In general, one could consider any $p$-form $A$, with $p=1, \ldots, k-1$, and write its action in terms of the field strength $F=d A$ as follows (using the notations of Ref.[22]):

$$
S=-\frac{1}{2 p !} \int F \wedge * F
$$

however, this is conformal invariant for $p=k-1$ only. For general $p$, this action is invariant under the gauge transformation $A \rightarrow A+d \mu$, with $\mu$ a $(p-1)$-form, thus we should introduce gauge-fixing terms and ghost fields. The ghost action is 
also gauge invariant, so we should consider ghosts of ghosts: the resulting tower of ghost fields contains all the forms of lower degree, till the 0-forms which have no further gauge invariance. This quantization problem is well understood [20] [22] and the resulting quantum action takes the form:

$$
S=-\frac{1}{2} \sum_{i=0}^{p} \frac{1}{(p-i) !} \int A_{p-i} \wedge * \Delta_{p-i}^{i+1} A_{p-i}
$$

where the $(p-i)$-form $A_{p-i}$ for $i$ even (odd) is a bosonic (fermionic) field and $\Delta_{p-i}$ is the Hodge-de Rham operator $\Delta=d \delta+\delta d$ expressed in terms of the exterior derivative $d$ and the coderivative $\delta$. It follows that the partition function for the antisymmetric tensor theory $(p=k-1)$ in $d=2 k$ is given by the following product of determinants:

$$
Z_{A T}=\frac{1}{\operatorname{det}^{1 / 2}\left(\Delta_{k-1}\right)} \cdot \frac{\operatorname{det}\left(\Delta_{k-2}\right)}{\operatorname{det}^{3 / 2}\left(\Delta_{k-3}\right)} \cdots\left(\frac{\operatorname{det}^{(k-1) / 2}\left(\Delta_{1}\right)}{\operatorname{det}^{k / 2}\left(\Delta_{0}\right)}\right)^{(-1)^{k-1}}
$$

On the geometry of the sphere, the eigenvalues and degeneracies for exact $(p+1)$ forms and coexact $p$-forms coincide [22], so we can simplify the ratios in the previous expression by restricting the determinants to the spectra of coexact forms:

$$
Z_{A T}=\left[\frac{1}{\operatorname{det}\left(\Delta_{k-1}^{c e}\right)} \cdot \frac{\operatorname{det}\left(\Delta_{k-2}^{c e}\right)}{\operatorname{det}\left(\Delta_{k-3}^{c e}\right)} \cdots\left(\frac{\operatorname{det}\left(\Delta_{1}^{c e}\right)}{\operatorname{det}\left(\Delta_{0}^{c e}\right)} V_{2 k}\right)^{(-1)^{k-1}}\right]^{1 / 2}
$$

where the superscript in $\Delta^{c e}$ means the restriction to coexact forms. Note the volume factor in the last term that arises from the regularization of the zero mode of the 0-form, which does not cancel out in the last ratio of Eq.(3.3).

The eigenvalues and degeneracies of the Hodge-de Rham operator on coexact $p$ forms in $S^{2 k}$ are given by 22 :

$$
\begin{aligned}
\lambda_{p, l} & =(l+p)(l+2 k-p-1) \\
\delta_{p, l} & =\frac{(l+2 k-1) !}{p !(2 k-p-1) !(l-1) !} \frac{2 l+2 k-1}{(l+p)(l+2 k-p-1)}
\end{aligned}
$$

The calculation of the trace anomaly thus involves the following alternate sum of zeta-functions:

$$
\frac{d}{d \alpha} \log Z_{A T} \equiv \zeta_{A T}(0)=\sum_{p=0}^{k-1}(-1)^{k-p-1} \zeta_{\Delta_{p}^{c e}}(0)+(-1)^{k-1} k .
$$


Each zeta-function can be analytically continued with the help of Eq. (2.8); after some simplifications, the result reads:

$$
\zeta_{A T}(0)=\sum_{q=1}^{k} \frac{(-1)^{q}}{(k-q) !(k+q-1) !} \sum_{i=1}^{k} \alpha_{i}(q) \sum_{m=0}^{i}(2 q-1)^{m} \frac{(i-1) !}{m !(i-m) !} B_{2 i-m},
$$

where the coefficients $\alpha_{i}(q)$, with $q=k-p$, are defined by the expression:

$$
\begin{aligned}
\frac{(\sigma+k) !}{(\sigma-k) !} & =\prod_{i=0}^{k-1}[(\sigma+q)(\sigma-q+1)-i(i+1)-q(1-q)] \\
& =\sum_{i=1}^{k} \alpha_{i}(q)[(\sigma+q)(\sigma-q+1)]^{i} .
\end{aligned}
$$

The result (3.7) can again be written in the compact form (using $B^{n} \rightarrow B_{n}$ ):

$$
\zeta_{A T}(0)=\sum_{q=1}^{k} \frac{(-1)^{q}}{(k-q) !(k+q-1) !} \int_{0}^{B(B+2 q-1)} \frac{d t}{t} \prod_{i=0}^{k-1}[t-i(i+1)-q(1-q)] .
$$

The first few values of this quantity for $d=\{4,6, \ldots, 12\}$ are:

$$
\zeta_{A T}(0)=\left\{-\frac{62}{90}, \frac{221}{210},-\frac{8051}{5670}, \frac{1339661}{748440},-\frac{525793111}{243243000}, \frac{3698905481}{1459458000}\right\} .
$$

For $k=2$ this matches the well-known trace anomaly of the vector field [15], and for $k=3$ it checks the recent result for the two-form gauge field in Ref. 23.

\section{Anomaly versus number of degrees of freedom}

The trace anomaly in $2 k$ dimensions contains the Euler density $G$ and a number of conformal covariant polynomials which involve the Weyl tensor $W$ and its derivatives, and have dimension $2 k$ [9]:

$$
\langle\Theta(x)\rangle=\lambda\left(a G(x)-c W(x) \Delta^{k-2} W(x)+O\left(W^{3}\right)+\ldots\right) .
$$

In this Equation, we called $a$ the coefficient of the Euler density $G$ and $c$ that of the term quadratic in the Weyl tensor, with $\Delta$ a conformal covariant Laplacian, and we left unspecified the other conformal covariant polynomials. The general form (4.1) can be integrated on conformally flat manifolds $\mathcal{M}$ to yield:

$$
\frac{d}{d \alpha} \log Z[\mathcal{M}]=-\frac{\lambda}{V_{2 k-1}} a \chi=\zeta_{\Delta}(0) \frac{\chi}{2}
$$


with $\chi=\int G$ the Euler characteristic. In the right part of this Equation, we also matched the general expression with the previous calculation on the sphere, for which $\chi\left(S^{2 k}\right)=2$. The normalization coefficient $\lambda$ remains to be chosen for the complete definition of $a$.

In the following, we shall argue that there exist an absolute, i.e. dimension and scale independent normalization for $a$ (call it $a=\hat{a}$ ), owing to the topological invariance of the Euler characteristic, and a relative, $d$-dependent normalization suitable for using $a$ as a counting function.

Let us discuss the first definition. The Euler characteristic $\chi(\mathcal{M})$ has a natural normalization because it takes integer values: it counts the number $b_{p}$ of non-trivial zero modes of the Hodge-De Rham operator $\Delta_{p}$ on the manifold $\mathcal{M}$, more precisely their alternating sum $b_{0}-b_{1}+b_{2}-\cdots+b_{2 k}$. On the other side of Eq.(4.2), the scale derivative of $\log Z$ is also a number free from ambiguities, being the finite part after regularization of the "number of positive modes" of a given Laplacian on $\mathcal{M}$. The ratio of these two numbers is an interesting dimension and scale independent quantity; thus, we identify it with $a=\hat{a} \equiv \zeta_{\Delta}(0)$, upon choosing the normalization $\lambda=-V_{2 k-1} / 2$.

This discussion suggests a similarity between the topological part of the trace anomaly and the chiral anomaly, which counts the number of certain zero modes on $\mathcal{M}$, as a consequence of the index theorem for the Dirac operator [11]. We have seen that the Laplacians of the free fields in Sections 2 and 3 do not have zero modes on the sphere, but nevertheless their trace anomaly is proportional to $\chi$ which counts zero modes. Suppose instead that $\Delta$ had $n$ zero modes: they would be regularized by an infra-red cut-off $\rho$ and would contribute to the partition function a factor $Z \sim \rho^{n}$, leading nicely to $\hat{a}=n$. For the actual $\Delta$ without zero modes, we can think that the ultra-violet regularization of the non-zero modes produces "effective" zero modes. In conclusion, $\hat{a}=\zeta_{\Delta}(0)$ can be interpreted as counting effective zero modes of the Laplacian of the theory.

We now discuss the dependence of this quantity on the dimension and the spin. Equation (2.13) shows some values of $\hat{a}$ for the scalar theory; one sees that their sign alternates with $k$ and that they decrease (exponentially) with the dimension. Actually, the large $d$ limit is equivalent to the semiclassical limit for the scalar theory, and the anomaly should go to zero. The semiclassical limit can be easily understood by defining the theory on a space-time lattice: the field variables are located on the 


\begin{tabular}{|c||c|c|c|c|c|c|c|c|}
\hline$d$ & 4 & 6 & 8 & 10 & 12 & 14 & & $2 k$ \\
\hline \hline$a(S)$ & 1 & 1 & 1 & 1 & 1 & 1 & & \\
\hline$a(F)$ & 11 & $\frac{191}{5}$ & $\frac{2497}{23}$ & $\frac{73985}{263}$ & $\frac{92427157}{133787}$ & $\frac{257184319}{157009}$ & $\cdots$ & \\
\hline$a(A T)$ & 62 & $\frac{3978}{5}$ & $\frac{161020}{23}$ & $\frac{13966610}{263}$ & $\frac{4466621324}{133787}$ & $\frac{3107080060404}{157009}$ & $\cdots$ & \\
\hline \hline$r(S)$ & 1 & 1 & 1 & 1 & 1 & 1 & & 1 \\
\hline$r(F)$ & 2.75 & 4.77 & 6.79 & 8.79 & 10.79 & 12.80 & $\cdots$ & $\simeq 2 k$ \\
\hline$r(A T)$ & 31 & 132.6 & 350.0 & 727.7 & 1310. & 2142. & $\cdots$ & $\simeq(2 k)^{3}$ \\
\hline
\end{tabular}

Table 1: Counting function $a$ and the corresponding weight per field component $r$ in various dimensions $d$, with asymptotic behaviours for $d \rightarrow \infty$.

lattice sites, and the discretized Laplacian of the field at one point $\Delta \phi\left(x_{o}\right)$ is the sum of all the $2 d$ field variables on the nearest neighbour sites. As the value of $d$ increases, the action approaches its mean-field approximation.

By the same argument we expect that the values of $\hat{a}$ for the fermion and antisymmetric tensor fields should also vanish for large $d$. This is indeed the case: we should take the values in Eqs.(2.19, 3.10) and divide them for the corresponding number of on-shell field components (number of independent polarizations), which are [20]:

$$
n(F)=2^{k}, \quad n(A T)=\frac{(2 k-2) !}{[(k-1) !]^{2}} .
$$

In conclusion, the trace anomaly $\hat{a}$ is a measure of effective zero modes and decreases for large $d$ in absolute units as expected.

We now analyse the coefficient $a$ as a measure of degrees of freedom in field theory; this interpretation would be implied by the eventual proof of the $c$-theorem. In this case, we should normalize $a(S)=1$ for the scalar field in any dimension, by choosing $\lambda$ in (4.2) accordingly. The values of $a$ in this normalization are reported in the first three lines of Table(1); the last three lines instead contain the relative weights per field component:

$$
r(\sigma)=\frac{a(\sigma)}{n(\sigma)}, \quad \sigma=S, F, A T .
$$

The last column in Table (1) gives their asymptotic behaviour for large dimension, which were determined numerically from Eqs. 2.12,2.18,3.9) (more precisely, $r(A T) \simeq$ 


\begin{tabular}{|c||c|c|c|c|c|c|c|c|}
\hline$d$ & 4 & 6 & 8 & 10 & 12 & 14 & & $2 k$ \\
\hline \hline$c(S)$ & 1 & 1 & 1 & 1 & 1 & 1 & & \\
\hline$c(F)$ & 6 & 20 & 56 & 144 & 352 & 832 & $\cdots$ & \\
\hline$c(A T)$ & 12 & 90 & 560 & 3150 & 16632 & 84084 & $\cdots$ & \\
\hline \hline$r^{\prime}(S)$ & 1 & 1 & 1 & 1 & 1 & 1 & & 1 \\
\hline$r^{\prime}(F)$ & $\frac{3}{2}$ & $\frac{5}{2}$ & $\frac{7}{2}$ & $\frac{9}{2}$ & $\frac{11}{2}$ & $\frac{13}{2}$ & $\cdots$ & $\simeq k$ \\
\hline$r^{\prime}(A T)$ & 6 & 15 & 28 & 45 & 66 & 91 & $\cdots$ & $\simeq 2 k^{2}$ \\
\hline
\end{tabular}

Table 2: Counting function $c$ and the corresponding weight per field component $r^{\prime}$ in various dimensions $d$, with asymptotic behaviours for $d \rightarrow \infty$.

$\left.0.943(2 k)^{3}\right)$. We find that these ratios do not go to one, as we would naively expect in the semiclassical limit, but grow, in particular for the antisymmetric tensor: already at $d=4$ the vector field has a rather large weight. Therefore, this "quantum" measure of degrees of freedom is always different from the classical number of field components - a fact that is rather counter-intuitive.

Apart from this physical interpretation, the behaviour of $a$ is not surprising because it also occurs for other terms in the trace and gravitational anomalies. This means that the spinful theories approach the semiclassical limit more slowly than the scalar theory, or that they interact more strongly with the background metric. Let us first discuss the other trace anomaly coefficient $c$ which was introduced in Eq. (4.1); this number also gives the normalization of the stress tensor correlator in flat space [16] and has been already computed in the Refs. [16][24] for the free theories considered here. In the same normalization $c(S)=1$, the results are:

$$
c(F)=2^{k-1}(2 k-1), \quad c(A T)=\frac{(2 k) !}{2[(k-1) !]^{2}}, \quad(d=2 k) .
$$

In Table (2) we display the first few values of $c$ as well as the corresponding weights per field component $r^{\prime}(\sigma)=c(\sigma) / n(\sigma)$. As we anticipated, these ratios grow with the dimension, although with milder asymptotic behaviours.

The same enhancement is observed in the chiral anomaly of pure gravitational origin, which occurs for dimensions $d=2+4 n$. The comprehensive study of Ref. [11] considers the following chiral fields: the Weyl fermion (one half of the Dirac fermion), 
the gravitino and the self-dual antisymmetric tensor (one half of that considered here). The complete expression of this chiral anomaly contains several independent terms, made by the different traces of the product of $(2+2 n)$ Riemann two-forms, and their coefficients span a wide range of values [11]. The larger coefficient is found for the genuine $d=2+4 n$ term, which is the $(n+1)$-th Pontryagin class $p_{n+1}(\mathcal{M})=$ $\operatorname{Tr}\left(R^{2 n+2}\right)+\cdots$.

We have computed the corresponding weights per field component for the three field types considered in Ref. [11] and for $d=6,10,14$ : these weights again grow with the dimension, the faster the higher spin value; moreover, the relative growth between the antisymmetric tensor and the fermion is much larger than the order $O\left(d^{2}\right)$ found for the coefficient $a$. The origin of this enhancement is not immediately apparent in the diagrammatic calculation of Ref.[1], because the coupling of spin to gravity does not have a standard group-theoretic form valid for all spin values. Nevertheless, the number of external legs in the anomalous loops increase with the dimension and the algebraic complexity enhances the spin dependence.

\section{Discussion}

Although puzzling, the measure of degrees of freedom given by a could be consistent with the renormalization group flow. Here we can check a couple of standard renormalization-group patterns and discuss a $c$-theorem conjecture [12] which is consistent with the observed enhancement.

A well-known flow is provided by a (generalized) gauge theory which has massive gauge fields in the infrared, together with massless scalars due to spontaneous symmetry breaking of a global symmetry; our results imply a very large value for $a_{U V}$, which better fulfills the $c$-theorem inequality $a_{U V}>a_{I R}$. Another example is the Higgs phenomenon, in which scalar degrees of freedom become extra components for the massive antisymmetric tensor. For large $d$, these extra components contribute to $a$ far less than the original components of the massless field: anyhow, no contradiction arises in going back to the UV limit, because the massless limit of the massive gauge theory is different from the massless theory.

In the Reference [12], it has been conjectured that the canonical form of the twodimensional $c$-theorem could extend to the class of higher dimensional theories char- 
acterized by $c=\nu a$, with $\nu(d)$ a given constant. The field theories described by the AdS/CFT correspondence belong to this class [13] [14]; for instance, the vector multiplet of $\mathcal{N}=4$ supersymmetry in four dimensions. In our notations $a(S)=c(S)=1$, the equation $c=\nu a$ of Ref. [12] reads:

$$
c-(2 k+1) \frac{\Gamma(2 k)}{\Gamma(k)^{2}}\left|\zeta_{\Delta_{0}}(0)\right| a=0, \quad(d=2 k) .
$$

Since the scalar, fermion and antisymmetric tensor fields contribute differently to $a$ and $c$, and with definite sign, there is no a-priori guarantee that this equation admits solutions in higher dimensions.

We can easily obtain the explicit form of this equation in each dimension by using the results in the two Tables; for $d=4,6,8,10$, we find:

$$
\begin{array}{rrlrl}
d=4: & 2 N_{S}+ & 7 N_{F}- & 26 N_{A T}=0 ; \\
d=6: & 13 N_{S}+ & 169 N_{F}- & 2358 N_{A T}=0 ; \\
d=8: & 67 N_{S}+ & 2543 N_{F}- & 110620 N_{A T}=0 ; \\
d=10: & 817 N_{S}+81535 N_{F}- & 9994610 N_{A T}=0 ;
\end{array}
$$

where $\left(N_{S}, N_{F}, N_{A T}\right)$ are the field multiplicities. We see that the dominance of $a(A T)$ yields a crucial minus sign in these equations, which ensures a solution in any dimension. Therefore, we have found that the behaviour of $a$ makes the $c$-theorem conjecture possible in any dimension.

The integer equations ( 5.2) has two independent solutions $\left(N_{S}, N_{F}, N_{A T}\right)$, which can be thought of as being vectors generating a two-dimensional sub-lattice of a threedimensional integer lattice. Some sample solutions, with minimal values for $N_{A T}$, are the following:

$$
\begin{aligned}
& d=4: \quad(6,2,1), \quad(13,0,1) \text {; } \\
& d=6: \quad(161,169,13), \quad(265,161,13) \text {; } \\
& d=8: \quad(835,65,2), \quad(2524,64,3) \text {; } \\
& d=10 \quad \text { : } \quad(11950,248,3), \quad(47095,141,5) \text {. }
\end{aligned}
$$

\section{Acknowledgments}

We thank D. Anselmi, A. Coste, R. Guida, N. Magnoli and A. Schwimmer for interesting discussions on the $c$-theorem and the trace anomaly. This work is supported in part by the European Community Network grant FMRX-CT96-0012. 


\section{References}

[1] A. B. Zamolodchikov, JETP Lett. 43 (1986) 730 .

[2] J. Cardy, Phys. Lett. B215 (1998) 749 .

[3] A. Cappelli, D. Friedan, J. I. Latorre, Nucl. Phys. B352 (1991) 616 .

[4] D. Anselmi, D. Z. Freedman, M.T. Grisaru and A.A. Johansen, Nucl. Phys. B526 (1998) 543 .

[5] D. Z. Freedman, S. S. Gubser, K. Pilch and N. P. Warner, hep-th/9904017 ;

L. Girardello, M. Petrini, M. Porrati and A. Zaffaroni, JHEP 9812 (1998) 022;

S.S. Gubser, Class. Quantum Grav. 17 (2000) 1081, hep-th/9910117 and Refs. therein .

[6] S. Forte and J. I. Latorre, Nucl. Phys. B535 (1998) 709 .

[7] D. Anselmi, Ann. Phys. 276 (1999) 361; Nucl. Phys. B567 (2000) 331, hep-th/9905005 .

[8] Osborn and J. I. Latorre, Nucl. Phys. B511 (1998) 737.

[9] S. Deser and A. Schwimmer, Phys. Lett. B309 (1993) 279 .

[10] S. W. Hawking, Commun. Math. Phys. 55 (1977) 149.

[11] L. Alvarez-Gaumé and E. Witten, Nucl. Phys. B234 (1984) 269.

[12] D. Anselmi, Phys. Lett. B476 (2000) 182, hep-th/9908014 .

[13] M. Henningson and K. Skenderis, JHEP 9807 (1998) 23.

[14] D. Anselmi, L. Girardello, M. Porrati and A. Zaffaroni, hep-th/0002066 .

[15] For a review see: N. D. Birrel and P .C. W. Davies, Quantum Fields in Curved Space, Cambridge Univ. Press (1982), Cambridge .

[16] A. Cappelli and A. Coste, Nucl. Phys. B314 (1989) 707 .

[17] E. J. Copeland and D. Toms, Class. Quantum Grav. 3 (1986) 431 .

[18] W. I. Weisberger, Nucl. Phys. B284 (1987) 171 . 
[19] P. Candelas and S. Weinberg, Nucl. Phys. B237 (1984) 397.

[20] P. K. Townsend, Phys. Lett. B88 (1979) 97; W. Siegel, Phys. Lett. B93 (1980) 170 ; D. Z. Freedman and P. K. Townsend, Nucl. Phys. B177 (1981) 282; T. Kimura, Prog. Theor. Phys. 65 (1981) 338 ; Yu. N. Obukhov, Phys. Lett. B109 (1982) 195 .

[21] G. W. Gibbons and P. K. Townsend, Phys. Rev. Lett. 71 (1983) 3754; D. M. Kaplan and J. Michelson, Phys. Rev. D53 (1996) 3474.

[22] E. J. Copeland and D. Toms, Nucl. Phys. B255 (1985) 201, and Refs. therein .

[23] F. Bastianelli, S. Frolov and A.A. Tseytlin, hep-th/0001041 .

[24] D. Anselmi, Class. Quantum Grav. 17 (2000) 1383, hep-th/9906167 . 